\title{
Author Correction: Challenging nationalism through science in 1919
}

Stephen Pompea and Pedro Russo

Correction to: Nature Astronomy https://doi.org/10.1038/s41550-019-0893-x, published online 19 September 2019.

In the version of this Comment originally published, in the fourth paragraph the date of the eclipse incorrectly read 20 May 1919; it should have been 29 May 1919. This has now been corrected.

Published online: 5 November 2019

https://doi.org/10.1038/s41550-019-0956-Z

๑) Springer Nature Limited 2019 